\title{
A Cost Comparison of the Synthetic and Shewhart $\bar{X}$ Charts
}

\author{
Wai Chung Yeong', Sok Li Lim², Zhi Lin Chong', Peh Sang Ng ${ }^{3}$ \\ ${ }^{1}$ Department of Operations and Management Information Systems, Faculty of Business and Accountancy \\ Universiti Malaya, 50603 Kuala Lumpur, Malaysia \\ yeongwc@um.edu.my \\ ${ }^{2}$ Institute of Mathematical Sciences, Faculty of Science, Universiti Malaya \\ 50603 Kuala Lumpur, Malaysia \\ sokli@um.edu.my \\ ${ }^{3}$ Department of Physical and Mathematical Sciences, Faculty of Science, Universiti Tunku Abdul Rahman \\ 31900 Kampar, Malaysia \\ chongzl@utar.edu.my,psng@utar.edu.my
}

\begin{abstract}
Although control charts are useful tools for quality monitoring, the cost of implementing the chart may prohibit practitioners from implementing it. The cost of sampling, cost of repairs, cost of defective products due to a failure in detecting out-of-control conditions, cost of false alarms etc can be prohibitively high. Hence, this paper compares the cost between the synthetic $\bar{X}$ and Shewhart $\bar{X}$ charts, so that practitioners could identify which chart is more economical to implement. The synthetic $\bar{X}$ chart was initially proposed to improve the ability in detecting changes in the process mean. This paper has shown that not only does the synthetic $\bar{X}$ chart has better detection ability than the Shewhart $\bar{X}$ chart, it is also more economical to implement. Thus, practitioners are recommended to adopt the synthetic $\bar{X}$ chart.
\end{abstract}

Keywords: Optimal Chart Parameters, Optimal Cost, Quality Control, Shewhart $\bar{X}$ Chart, Synthetic $\bar{X}$ Chart.

\section{Introduction}

Control charts are frequently adopted to monitor various processes to detect the presence of assignable causes which results in an out-of-control condition, especially in various engineering applications and manufacturing environments. However, the cost of implementing control charts can be high, which makes it less attractive for practitioners to adopt it. In the literature, control charts are mostly designed to optimize its' performance in detecting an out-of-control condition, but the cost of implementing the chart is not given much attention, even though cost is very important for industries.

Hence, this paper would like to compare the cost of two charts in the literature, i.e. the Shewhart $\bar{X}$ chart and the synthetic $\bar{X}$ chart. The Shewhart $\bar{X}$ chart is the first chart proposed in the literature. This chart works by producing an outof-control signal when the sample mean is outside the upper or lower control limits, i.e. when $\bar{X}>U C L$ or $\bar{X}<L C L$. Due to its' simplicity, it is frequently implemented in industries. However, it shows weak performance in detecting small and moderate shifts.

Since then, a number of new charts are proposed which improves the performance of the Shewhart $\bar{X}$ chart, one of which is the synthetic $\bar{X}$ chart. The synthetic $\bar{X}$ chart is proposed by [1]. The synthetic chart consists of a $\bar{X} / S$ sub-chart and a $C R L / S$ sub-chart. It operates by defining an $\bar{X}$ sample as non-conforming if the sample mean, $\bar{X}$ is smaller than the lower control limit of the $\bar{X} / S$ sub-chart or larger than the upper control limit of the $\bar{X} / S$ sub-chart. An out-of-control signal is generated when the number of $\bar{X}$ samples between the current and the last non-conforming samples, i.e. the conforming run length, is smaller than or equal to $L$, the lower control limit of the $C R L / S$ sub-chart. Note that the conforming run length follows a geometric distribution. [1] has shown that the synthetic chart shows significantly better performance than the Shewhart chart, in terms of fewer samples required to detect an out-of-control condition. Several extensions are done on the synthetic chart. [2] proposed a synthetic chart to jointly monitor the mean and variance, [3, 4] proposed a 
synthetic chart to monitor the process mean for skewed distributions and [5] proposed a synthetic chart to monitor the coefficient of variation. Section 2 gives a brief review of the Shewhart $\bar{X}$ and synthetic $\bar{X}$ charts.

In this paper, we would like to compare the cost of implementing the Shewhart $\bar{X}$ and synthetic $\bar{X}$ charts. The economic model by [6] is adopted to obtain the optimal cost. Section 3 gives a review of the economic model. This is followed by Section 4 which compares the cost based on a number of examples. Finally, concluding remarks are given in Section 5.

\section{Shewhart $\bar{X}$ and Synthetic $\bar{X}$ Charts}

This section gives a review of the Shewhart $\bar{X}$ and synthetic $\bar{X}$ charts. This section shows how the charts operate and gives the formulae to compute the Average Run Length $(A R L)$. Note that the $A R L$ allows practitioners to calculate the longrun average number of samples until the chart signals an out-of-control condition. The $A R L$ is often adopted as a performance measure.

\subsection{Shewhart $\bar{X}$ Chart}

The Shewhart $\bar{X}$ chart is the most widely used chart to monitor the process mean in industries. It consists of three lines, i.e. the upper control limit $(U C L)$, the center line $(C L)$, and the lower control limit $(L C L)$. When the mean of the quality characteristic being monitored is larger than the $U C L$, or smaller than the $L C L$, the $\bar{X}$ chart will signal an out-of-control condition.

In constructing the $\bar{X}$ chart, the quality characteristic is assumed to be normally distributed with mean $\mu$ and standard deviation $\sigma$, where both $\mu$ and $\sigma$ are known. If $X_{1}, X_{2}, \ldots, X_{n}$ is a sample of size $n$ from the $N\left(\mu, \sigma^{2}\right)$ distribution, then the sample mean is calculated as

$$
\bar{X}=\frac{X_{1}+X_{2}+\ldots+X_{n}}{n}
$$

where $\bar{X}$ is normally distributed with mean $\mu$ and standard deviation $\sigma$. The limits of the Shewhart $\bar{X}$ chart are computed as

$$
U C L=\mu_{0}+k \frac{\sigma}{\sqrt{n}}
$$

And

$$
L C L=\mu_{0}-k \frac{\sigma}{\sqrt{n}}
$$

where $k$ is a constant controlling the width of the control limits of the $\bar{X}$ chart, while $\mu$ and $\sigma$ are the in-control mean and standard deviation, respectively. Note that $\mu_{0}$ is used as the center line.

It is assumed that the quality characteristic is normally distributed. However, even if the quality characteristic is not normally distributed, the above results are still approximately correct, due to the central limit theorem [7].

The average number of samples required to signal a process mean shift from the in-control mean $\mu_{0}$ to the out-ofcontrol mean $\mu_{1}=\mu_{0} \pm \delta \sigma$ is called the average run length denoted as $A R L_{1}$ and is widely used to assess the performance of control charts. For the $\bar{X}$ chart, $A R L_{1}$ is calculated as [7]

$$
A R L_{1}=\frac{1}{1-\beta}
$$


where $\beta=\Phi(k-\delta \sqrt{n})-\Phi(-k-\delta \sqrt{n})$ and $\Phi($.$) denotes the cumulative distribution function of the standard normal$ distribution. The in-control $A R L, A R L_{0}$, can be calculated by letting $\delta=0$. Hence, $A R L_{0}$ is computed as

$$
A R L_{0}=\frac{1}{2 \Phi(-k)}
$$

Note that $A R L_{0}$ is the average number of samples taken until the first false alarm is signaled.

\subsection{Synthetic $\bar{X}$ Chart}

[1] proposed a synthetic chart that makes the $\bar{X}$ chart and the conforming run length $(C R L)$ chart work together. The synthetic chart consists of a $\bar{X} / S$ sub-chart and a $C R L / S$ sub-chart. The steps to implement a synthetic chart are as follows [1]:

1. Determine the lower and upper control limits, $L C L_{\bar{X} / S}$ and $U C L_{\bar{X} / S}$, of the $\bar{X} / S$ sub-chart and the lower control limit, $L$, of the $C R L / S$ sub-chart, where

$$
L C L_{\bar{X} / S}=\mu_{0}-k \sigma_{\bar{X}}
$$

and

$$
U C L_{\bar{X} / S}=\mu_{0}+k \sigma_{\bar{X}}
$$

where $\mu_{0}$ is the in-control process mean and $\sigma_{\bar{X}}$ is the standard deviation of the sample mean.

2. At each sampling point, a random sample of $n$ observations is taken and the sample mean, $\bar{X}$ is calculated.

3. If $\bar{X}$ is larger than $L C L_{\bar{X} / S}$ and smaller than $U C L_{\bar{X} / S}$, then the sample is declared as "conforming" and the control flow returns to Step 2. Otherwise, the sample is declared as "non-conforming" and the control flow moves to the next step.

4. Count the number of $\bar{X}$ samples between the current (included in the count) and the last (excluded in the count) nonconforming samples. This number is taken as the $C R L$ value of the $C R L / S$ sub-chart.

5. If the $C R L$ value is larger than $L$, the process is in-control and the control flow returns to Step 2. Otherwise, an outof-control signal will be generated and actions are taken to find and remove the assignable cause(s) so that the process returns to an in-control state, after which the control flow returns to Step 2.

Note that unlike the Shewhart $\bar{X}$ chart, an out-of-control signal is not generated when a sample value of $\bar{X}$ falls beyond the control limits of the $\bar{X} / S$ sub-chart. Instead, the chart just indicates a nonconforming sample. The out-of-control signal is only generated when the $C R L$ value of the $C R L / S$ sub-chart is smaller than or equal to the lower control limit $(L)$ of the $C R L / S$ sub-chart. The $A R L_{1}$ of the synthetic $\bar{X}$ chart is calculated as [1]

$$
A R L_{1}=\frac{1}{P} \times \frac{1}{1-(1-P)^{L}},
$$

where $P=1-\Phi(k-\delta \sqrt{n})+\Phi(-k-\delta \sqrt{n})$ and $\Phi($.$) denotes the standard normal cumulative distribution function. The$ $A R L_{0}$ is computed as 


$$
A R L_{0}=\frac{1}{2 \Phi(-k)} \times \frac{1}{1-[1-2 \Phi(-k)]^{L}} .
$$

\section{Economic Model of Control Charts}

This section shows the economic model to obtain the optimal chart parameters which minimizes the cost. The cost of implementing the control chart is computed based on the cost function. It is assumed that the process starts in a state of control and follows a normal distribution with mean $\mu_{0}$ and standard deviation $\sigma$. A single assignable cause occurs at a random time and causes a shift in the process mean of a known magnitude $\delta \sigma$ so that the mean value is $\mu_{1}=\mu_{0} \pm \delta \sigma$. The occurrence of the assignable cause indicates that the process has gone out-of-control. The time until the assignable cause occurs is assumed to be exponentially distributed with rate $\lambda$. The process stays at the out-of-control level until a lack of control is indicated by the synthetic chart and adjustments are made to bring it back to the in-control level $\left(\mu=\mu_{0}\right)$. By using the approximation in [8], the expected cost per unit time, $C$, is obtained as:

$$
C=\left(\frac{C_{0}}{\lambda}+C_{1} B+\frac{b+c n}{h}\left(\frac{1}{\lambda}+B\right)+\frac{s Y}{A R L_{0}}+W\right) \div\left(\frac{1}{\lambda}+\frac{\left(1-\gamma_{1}\right) s T_{0}}{A R L_{0}}+E H\right),
$$

where $B=\left(A R L_{1}-0.5\right) h+F$,

$$
\begin{aligned}
& F=n e+\gamma_{1} T_{1}+\gamma_{2} T_{2}, \\
& s=\frac{1}{\lambda h}-0.5, \\
& E H=\left(A R L_{1}-0.5\right) h+G,
\end{aligned}
$$

and

$$
G=n e+T_{1}+T_{2} \text {. }
$$

The notations in Eq. (10) are defined as follows:

$b \quad$ Fixed cost per sample

c $\quad$ Cost per unit sampled

$C_{0} \quad$ Expected quality cost per unit time while in control

$C_{1} \quad$ Expected quality cost per unit time while out-of-control

$e \quad$ Expected time to sample and interpret one unit

$h \quad$ Sampling interval

$n \quad$ Sample size

$s \quad$ Expected number of samples taken before an assignable cause occur

$T_{0} \quad$ Expected search time for a false alarm

$T_{1} \quad$ Expected time to find the assignable cause

$T_{2} \quad$ Expected time to repair the process

$W \quad$ Cost of finding and fixing an assignable cause

$Y \quad$ Cost of false alarm

$\gamma_{1}=1$ if production continues during search

$=0$ if production stops during search

$\gamma_{2}=1$ if production continues during repair

$=0$ if production stops during repair

$\lambda$ Process failure rate

$\tau \quad$ Expected time of occurrence of the assignable cause 
The expected cost per unit time in Eq. (10) is derived from dividing the expected cost per cycle by the expected cycle length, where a cycle is defined as the start of successive in-control periods. The cycle time consists of the in-control period and out-of-control period, which consists of the time to signal, the time to sample and interpret the result, and the time to repair the process. The total cost in a cycle includes the cost due to nonconformities produced while in the in-control and out-of-control states, the cost of sampling, cost of false alarms, and cost of repairs.

The optimal chart parameters are the chart parameters which minimizes the cost. For the Shewhart $\bar{X}$ chart, this refers to the $(n, k, h)$ combination which results in minimum cost, while for the Synthetic $\bar{X}$ chart, this refers to the $(n, L, k, h)$ combination which results in minimum cost. Note that $h$ is the sampling interval. To obtain the optimal $h$, the partial derivative of $C$ with respect to $h$ is set to be equal to zero. This yields

$$
r_{1} h^{2}+r_{2} h+r_{3}=0
$$

where

$$
\begin{aligned}
& r_{1}=\frac{\left(A R L_{1}-0.5\right)\left[\lambda\left(Y+C_{1} T_{0}\left(-1+\gamma_{1}\right)\right)-2 A R L_{0}\left[C_{0}+\lambda\left(\left(A R L_{1}-0.5\right) b+\left(A R L_{1}-0.5\right) c n+W\right)+C_{1}(-1+F \lambda-G \lambda)\right]\right]}{2 \lambda A R L_{0}}, \\
& r_{2}=-\frac{2\left(A R L_{1}-0.5\right)\left[Y+C_{1} T_{0}\left(-1+\gamma_{1}\right)+A R L_{0}(b+c n)(1+F \lambda)\right]}{\lambda A R L_{0}}, \\
& r_{3}=-\frac{1}{2 \lambda^{2} A R L_{0}}\left[2 Y+2 C_{0} T_{0}\left(-1+\gamma_{1}\right)-b T_{0} \lambda-2\left(A R L_{1}-0.5\right) b T_{0} \lambda-2 C_{1} F T_{0} \lambda-c n T_{0} \lambda-2\left(A R L_{1}-0.5\right) c n T_{0} \lambda-2 T_{0} W \lambda+2 G Y \lambda+b T_{0} \gamma_{1} \lambda+2\left(A R L_{1}-0.5\right) b T_{0} \gamma_{1} \lambda+\right. \\
& \left.2 C_{1} F T_{0} \gamma_{1} \lambda+c n T_{0} \gamma_{1} \lambda+2\left(A R L_{1}-0.5\right) c n T_{0} \gamma_{1} \lambda+2 T_{0} W \gamma_{1} \lambda-b F T_{0} \lambda^{2}-c F n T_{0} \lambda^{2}+b F T_{0} \gamma_{1} \lambda^{2}+c F n T_{0} \gamma_{1} \lambda^{2}+2 A R L_{0}(b+c n)(1+F \lambda)(1+G \lambda)\right]
\end{aligned}
$$

The derivation of Eq. (11) is shown in [6]. The $h$ which solves Eq (11) is shown as follows:

$$
h=\frac{-r_{2}+\sqrt{r_{2}^{2}-4 r_{1} r_{3}}}{2 r_{1}} .
$$

To obtain the optimal $(n, k)$ and $(n, L, k)$, we consider values of $L$ within $\{1,2, \ldots, 20\}$, the values of $n$ between 0 and $\frac{40}{\delta^{2}}$ ([8] also considers the upper bound of $n$ as $\frac{40}{\delta^{2}}$ ), and values of $k$ within $\{0.01,0.02, \ldots, 3.00\}$. Note that only integer values of $n$ are used. For all combinations within this range, the optimal $h$ is computed from Eq. (12). Subsequently, the cost of all possible combinations of $(n, k, h)$ (for the Shewhart $\bar{X}$ chart) and $(n, L, k, h)$ (for the synthetic $\bar{X}$ chart) is calculated from Eq. (10). The combination with the smallest cost will be the optimal chart parameters, and its resulting cost is the minimum cost.

\section{Comparisons}

This section compares the optimal cost for the Shewhart $\bar{X}$ and synthetic $\bar{X}$ charts. The same example in [6] is adopted to illustrate the cost of implementing these two charts. This example is based on a foundry operation with a production rate of 84 castings per hour on one of its lines. A standard is set to prevent high carbon-silicate content as it will result in low tensile strength, which is an important product attribute. The cost and process parameters are $\lambda=0.02, \delta=0.86, e=T_{0}=T_{1}=0.083, T_{2}=0.75, \gamma_{1}=1, \gamma_{2}=0, C_{0}=\$ 114.24, C_{1}=\$ 949.20, Y=W=\$ 977.40, b=\$ 0$, $c=\$ 4.22$. By adopting the methodology in Section 3, the optimal cost for the Shewhart $\bar{X}$ and synthetic $\bar{X}$ charts are $\$ 206.84$ and $\$ 192.96$, respectively. This shows that the Shewhart $\bar{X}$ chart results in an increase in cost of $7.19 \%$ compared to the synthetic $\bar{X}$ chart.

To compare the cost of the Shewhart $\bar{X}$ and synthetic $\bar{X}$ charts based on more examples, a "one-factor-at-a-time" approach is adopted, where each time, one of the selected input parameters is made to vary around its actual value, while the 
other input parameters remain unchanged. For example, the actual value for $\lambda$ is 0.02 , but we consider the cases where $\lambda$ is 0.01 and 0.04 . Table 1 shows the values of the input parameters for the numerical examples adopted in this study.

Table 1: Input parameters for the numerical examples.

\begin{tabular}{|c|c|c|c|c|c|c|c|c|c|c|c|c|c|c|}
\hline No. & $\lambda$ & $\delta$ & $C_{0}(\$)$ & $C_{1}(\$)$ & $Y(\$)$ & $W(\$)$ & $b(\$)$ & $c(\$)$ & $e$ & $T_{0}$ & $T_{1}$ & $T_{2}$ & $\gamma_{1}$ & $\gamma_{2}$ \\
\hline 1 & .01 & .86 & 4.24 & 949.2 & 977.4 & 977.4 & 0 & 4.22 & 0.083 & 0.083 & 0.083 & 0.75 & 1 & 0 \\
\hline 2 & 0.02 & 0.86 & 14.24 & 949.2 & 977.4 & 977.4 & 0 & 4.22 & 0.083 & 0.083 & 0.083 & 0.75 & 1 & 0 \\
\hline 3 & 0.04 & 0.86 & 114.24 & 949.2 & 977.4 & 977.4 & 0 & 4.22 & 0.083 & 0.083 & 0.083 & 0.75 & 1 & 0 \\
\hline 4 & 0.02 & 0.5 & 114.24 & 949.2 & 977.4 & 977.4 & 0 & 4.22 & 0.083 & 0.083 & 0.083 & 0.75 & 1 & 0 \\
\hline 5 & 0.02 & 0.86 & 114.24 & 949.2 & 977.4 & 977.4 & 0 & 4.22 & 0.083 & 0.083 & 0.083 & 0.75 & 1 & 0 \\
\hline 6 & 0.02 & 1 & 114.24 & 949.2 & 977.4 & 977.4 & 0 & 4.22 & 0.083 & 0.083 & 0.083 & 0.75 & 1 & 0 \\
\hline 7 & 0.02 & 2 & 114.24 & 949.2 & 977.4 & 977.4 & 0 & 4.22 & 0.083 & 0.083 & 0.083 & 0.75 & 1 & 0 \\
\hline 8 & 0.02 & 0.86 & 57.12 & 949.2 & 977.4 & 977.4 & 0 & 4.22 & 0.083 & 0.083 & 0.083 & 0.75 & 1 & 0 \\
\hline 9 & 0.02 & 0.86 & 114.24 & 949.2 & 977.4 & 977.4 & 0 & 4.22 & 0.083 & 0.083 & 0.083 & 0.75 & 1 & 0 \\
\hline 10 & 0.02 & 0.86 & 228.48 & 949.2 & 977.4 & 977.4 & 0 & 4.22 & 0.083 & 0.083 & 0.083 & 0.75 & 1 & 0 \\
\hline 11 & 0.02 & 0.86 & 114.24 & 474.6 & 977.4 & 977.4 & 0 & 4.22 & 0.083 & 0.083 & 0.083 & 0.75 & 1 & 0 \\
\hline 12 & 0.02 & 0.86 & 114.24 & 949.2 & 977.4 & 977.4 & 0 & 4.22 & 0.083 & 0.083 & 0.083 & 0.75 & 1 & 0 \\
\hline 13 & 0.02 & 86 & 114.24 & 1898.4 & 977.4 & 977.4 & 0 & 4.22 & 83 & 0.083 & .083 & 0.75 & 1 & 0 \\
\hline 14 & 0.02 & 0.86 & 114.24 & 949.2 & 488.7 & 977.4 & 0 & 4.22 & 0.083 & 0.083 & 0.083 & 0.75 & 1 & 0 \\
\hline 15 & 0.02 & 0.86 & 114.24 & 949.2 & 977.4 & 977.4 & 0 & 4.22 & 0.083 & 0.083 & 0.083 & 0.75 & 1 & 0 \\
\hline 16 & 0.02 & .86 & 114.24 & 949.2 & 1954.8 & 977.4 & 0 & 4.22 & 0.083 & 0.083 & 0.083 & 0.75 & 1 & 0 \\
\hline 17 & 0.02 & 86 & 114.24 & 949.2 & 977.4 & 488.7 & 0 & 4.22 & & 83 & 083 & 0.75 & 1 & 0 \\
\hline 18 & 0.02 & 0.86 & 114.24 & 949.2 & 977.4 & 977.4 & 0 & 4.22 & 0.083 & 0.083 & 0.083 & 0.75 & 1 & 0 \\
\hline 19 & 0.02 & 0.86 & 114.24 & 949.2 & 977.4 & 1954.8 & 0 & 4.22 & 0.083 & 0.083 & 0.083 & 0.75 & 1 & 0 \\
\hline 20 & 0.02 & 86 & 114.24 & 949.2 & 977.4 & 977.4 & $\mathbf{0}$ & 4.22 & 0.083 & 0.083 & 0.083 & 0.75 & 1 & 0 \\
\hline 21 & 0.02 & 0.86 & 114.24 & 949.2 & 977.4 & 977.4 & 5 & 4.22 & 0.083 & 0.083 & 0.083 & 0.75 & 1 & 0 \\
\hline 22 & 0.02 & 0.86 & 114.24 & 949.2 & 977.4 & 977.4 & 10 & 4.22 & 0.083 & 0.083 & 0.083 & 0.75 & 1 & 0 \\
\hline 23 & 0.02 & 0.86 & 114.24 & 949.2 & 977.4 & 977.4 & 0 & 2.11 & 0.083 & 0.083 & 0.083 & 0.75 & 1 & 0 \\
\hline 24 & 0.02 & 0.86 & 114.24 & 949.2 & 977.4 & 977.4 & 0 & 4.22 & 83 & 0.083 & 0.083 & 0.75 & 1 & 0 \\
\hline 25 & 0.02 & 0.86 & 114.24 & 949.2 & 977.4 & 977.4 & 0 & 8.44 & & 0.083 & 0.083 & 0.75 & 1 & 0 \\
\hline 26 & 0.02 & 0.86 & 114.24 & 949.2 & 977.4 & 977.4 & 0 & 4.22 & 0.042 & 0.083 & 0.083 & 0.75 & 1 & 0 \\
\hline 27 & 0.02 & .86 & 114.24 & 949.2 & 977.4 & 977.4 & 0 & 4.22 & $\mathbf{0 . 0 8 3}$ & 0.083 & 0.083 & 0.75 & 1 & 0 \\
\hline 28 & 0.02 & 0.86 & 114.24 & 949.2 & 977.4 & 977.4 & 0 & 4.22 & 0.166 & 0.083 & 0.083 & 0.75 & 1 & 0 \\
\hline 29 & 0.02 & 0.86 & 114.24 & 949.2 & 977.4 & 977.4 & 0 & 4.22 & 0.083 & 0.042 & 0.083 & 0.75 & 1 & 0 \\
\hline 30 & 0.02 & 0.86 & 114.24 & 949.2 & 977.4 & 977.4 & 0 & 4.22 & 0.083 & 0.083 & 0.083 & 0.75 & 1 & 0 \\
\hline 31 & 0.02 & 0.86 & 114.24 & 949.2 & 977.4 & 977.4 & 0 & 4.22 & 0.083 & 0.166 & 0.083 & 0.75 & 1 & 0 \\
\hline 32 & 0.02 & 0.86 & 114.24 & 949.2 & 977.4 & 977.4 & 0 & 4.22 & 0.083 & 0.083 & 0.042 & 0.75 & 1 & 0 \\
\hline 33 & 0.02 & 0.86 & 114.24 & 949.2 & 977.4 & 977.4 & 0 & 4.22 & 0.083 & 0.083 & 0.083 & 0.75 & 1 & 0 \\
\hline 34 & 0.02 & 0.86 & 114.24 & 949.2 & 977.4 & 977.4 & 0 & 4.22 & 0.083 & 0.083 & 0.166 & 0.75 & 1 & 0 \\
\hline 35 & 0.02 & 0.86 & 114.24 & 949.2 & 977.4 & 977.4 & 0 & 4.22 & 0.083 & 0.083 & 0.083 & 0.375 & 1 & 0 \\
\hline 36 & 0.02 & 0.86 & 114.24 & 949.2 & 977.4 & 977.4 & 0 & 4.22 & 0.083 & 0.083 & 0.083 & 0.75 & 1 & 0 \\
\hline 37 & 0.02 & 0.86 & 114.24 & 949.2 & 977.4 & 977.4 & 0 & 4.22 & 0.083 & 0.083 & 0.083 & 1.5 & 1 & 0 \\
\hline 38 & 0.02 & 0.86 & 114.24 & 949.2 & 977.4 & 977.4 & 0 & 4.22 & 0.083 & 0.083 & 0.083 & 0.75 & 1 & $\mathbf{0}$ \\
\hline 39 & 0.02 & 0.86 & 114.24 & 949.2 & 977.4 & 977.4 & 0 & 4.22 & 0.083 & 0.083 & 0.083 & 0.75 & $\mathbf{0}$ & 0 \\
\hline 40 & 0.02 & 0.86 & 114.24 & 949.2 & 977.4 & 977.4 & 0 & 4.22 & 0.083 & 0.083 & 0.083 & 0.75 & $\mathbf{0}$ & 1 \\
\hline 41 & 0.02 & 0.86 & 114.24 & 949.2 & 977.4 & 977.4 & 0 & 4.22 & 0.083 & 0.083 & 0.083 & 0.75 & 1 & 7 \\
\hline
\end{tabular}


Note that the values in bold for Table 1 are the values which are made to vary. Next, Table 2 shows the cost of the synthetic $\bar{X}$ and Shewhart $\bar{X}$ charts based on the numerical examples in Table 1 .

Table 2: Optimal cost of the synthetic $\bar{X}$ and Shewhart $\bar{X}$ charts.

\begin{tabular}{|c|c|c|}
\hline No. & Synthetic $\bar{X}$ Chart & Shewhart $\bar{X}$ Chart \\
\hline 1 & 165.00 & $174.48(5.75 \%)$ \\
\hline 2 & 192.96 & $206.84(7.19 \%)$ \\
\hline 3 & 237.23 & $257.11(8.38 \%)$ \\
\hline 4 & 230.86 & $248.60(7.68 \%)$ \\
\hline 5 & 192.96 & $206.84(7.19 \%)$ \\
\hline 6 & 184.99 & $197.55(6.79 \%)$ \\
\hline 7 & 159.86 & $167.08(4.52 \%)$ \\
\hline 8 & 139.16 & $153.67(10.43 \%)$ \\
\hline 9 & 192.96 & $206.84(7.19 \%)$ \\
\hline 10 & 300.35 & $312.92(4.19 \%)$ \\
\hline 11 & 168.38 & $176.21(4.65 \%)$ \\
\hline 12 & 192.96 & $206.84(7.19 \%)$ \\
\hline 13 & 229.55 & $252.59(10.04 \%)$ \\
\hline 14 & 189.08 & $200.54(6.06 \%)$ \\
\hline 15 & 192.96 & $206.84(7.19 \%)$ \\
\hline 16 & 196.66 & $212.87(8.24 \%)$ \\
\hline 17 & 183.76 & $197.75(7.61 \%)$ \\
\hline 18 & 192.96 & $206.84(7.19 \%)$ \\
\hline 19 & 211.36 & $225.02(6.46 \%)$ \\
\hline 20 & 192.96 & $206.84(7.19 \%)$ \\
\hline 21 & 195.77 & $209.03(6.77 \%)$ \\
\hline 22 & 198.30 & $211.06(6.43 \%)$ \\
\hline 23 & 181.40 & $194.38(7.16 \%)$ \\
\hline 24 & 192.96 & $206.84(7.19 \%)$ \\
\hline 25 & 208.07 & $222.75(7.06 \%)$ \\
\hline 26 & 188.12 & $200.14(6.39 \%)$ \\
\hline 27 & 192.96 & $206.84(7.19 \%)$ \\
\hline 28 & 200.76 & $217.19(8.18 \%)$ \\
\hline 29 & 192.96 & $206.84(7.19 \%)$ \\
\hline 30 & 192.96 & $206.84(7.19 \%)$ \\
\hline 31 & 192.96 & $206.84(7.19 \%)$ \\
\hline 32 & 192.36 & $206.26(7.23 \%)$ \\
\hline 33 & 192.96 & $206.84(7.19 \%)$ \\
\hline 34 & 194.16 & $208.01(7.13 \%)$ \\
\hline 35 & 194.33 & $208.30(7.19 \%)$ \\
\hline 36 & 192.96 & $206.84(7.19 \%)$ \\
\hline 37 & 190.27 & $204.00(7.22 \%)$ \\
\hline 38 & 192.96 & $206.84(7.19 \%)$ \\
\hline 39 & 191.35 & $205.18(7.23 \%)$ \\
\hline 40 & 205.01 & $218.69(6.67 \%)$ \\
\hline 41 & 206.63 & $220.37(6.65 \%)$ \\
\hline
\end{tabular}


From Table 2, it can be observed that for all the cases, the synthetic $\bar{X}$ chart has a lower cost than the Shewhart $\bar{X}$ chart. The value in parenthesis for Table 2 denotes the percentage of savings in cost when the synthetic $\bar{X}$ chart is used. The percentage of savings ranges from $4.19 \%$ to $10.43 \%$. Hence, it can be concluded that implementing the synthetic $\bar{X}$ chart is more economical than the Shewhart $\bar{X}$ chart.

\section{Conclusion}

This paper compares the cost of implementing the Shewhart $\bar{X}$ and synthetic $\bar{X}$ charts. This paper shows the background and the steps to implement these charts, as well as the cost function and the optimization algorithm to obtain the optimal chart parameters and cost. The comparison is based on a number of numerical examples. In all these examples, it was shown that the synthetic $\bar{X}$ chart shows lower cost than the Shewhart $\bar{X}$ chart. Hence, besides having better detection abilities, the synthetic $\bar{X}$ chart is also more economical to implement. Furthermore, the synthetic $\bar{X}$ chart is also easy to implement. Thus, practitioners are encouraged to adopt the synthetic $\bar{X}$ chart to monitor the quality characteristic of a process.

\section{References}

[1] Z. Wu and T. A. Spedding, "A synthetic control chart for detecting small shifts in the process mean," Journal of Quality Technology, vol. 32, pp. 32-38, 2000.

[2] A. F. B. Costa and M. A. Rahim, "A synthetic control chart for monitoring the process mean and variance," Journal of Quality in Maintenance Engineering, vol. 12, pp. 81-88, 2006.

[3] M. B. C. Khoo, Z. Wu and A. M. A. Atta, "A synthetic control chart for monitoring the process mean of skewed populations based on the weighted variance method," International Journal of Reliability, Quality and Safety Engineering, vol. 15, pp. 217-245, 2008.

[4] P. Castagliola, M. B. C. Khoo, "A synthetic scaled weighted variance control chart for monitoring the process mean of skewed populations," Communications in Statistics-Simulation and Computation, vol. 38, pp. 1659-1674, 2009.

[5] M. E. Calzada and S. M. Scariano, "A synthetic control chart for the coefficient of variation," Journal of Statistical Computation and Simulation, vol. 83, pp. 853-867, 2013.

[6] W. C. Yeong, M. B. C. Khoo, Z. Wu and P. Castagliola, "Economically optimum design of a synthetic $\bar{X}$ chart," Quality and Reliability Engineering International, vol. 28, pp. 725-741, 2012.

[7] D. C. Montgomery, Statistical Quality Control: A Modern Introduction. New York: Wiley \& Sons, 2009.

[8] K. J. Chung, "A simplified procedure for the economic design of $\bar{X}$-charts," International Journal of Production Research, vol. 20, pp. 1239-1246, 1990. 